\title{
Uso de dispositivos intravaginales con progesterona en vaquillonas para producción de carne: efecto del rango horario en que se realiza la inseminación artificial a tiempo fijo
}

\author{
USE OF INTRAVAGINAL DEVICES WITH PROGESTERONE IN BEEF HEIFERS: EFFECT OF THE TIME \\ RANGE IN WHICH FIXED TIMED ARTIFICIAL INSEMINATION IS PERFORMED
}

Jorge Monteserin ${ }^{2}$, Ricardo Chayer ${ }^{2}$, Jorge Cabodevila ${ }^{2}$, Santiago Callejas ${ }^{1,3}$

\section{Resumen}

Se utilizaron 333 vaquillonas para producción de carne con 15 meses de edad y condición corporal promedio de 6 (escala 1-9). El 53.5\% presentó un cuerpo lúteo activo al inicio del trabajo. En el día 0, se colocó un dispositivo intravaginal con $0.5 \mathrm{~g}$ de progesterona (DISP) y se inyectó $2 \mathrm{mg}$ de benzoato de estradiol. El día 8 se retiró el DISP y se inyectó $0.5 \mathrm{mg}$ de cloprostenol y $0.5 \mathrm{mg}$ de cipionato de estradiol i.m. El día $10 \mathrm{se}$ realizó la inseminación artificial a tiempo fijo (IATF), distribuyéndose los animales al azar para ser inseminados en tres rangos horarios: I) 48-50 h; II: $>50-53 \mathrm{~h}$; III) $>53-56 \mathrm{~h}$. Se utilizó semen congelado en pajuelas de $0.5 \mathrm{ml}$. El diagnóstico de gestación se realizó mediante ultrasonografía a los 30 días pos-IATF. Dos animales perdieron el DISP y fueron excluidos del análisis. No se observó efecto del rango horario en que se realizó la IATF luego de retirado el DISP sobre el porcentaje de preñez (I: 64.0\%; II: 69.8\%; III: $66.9 \%$ ). Se concluye que en vaquillonas para carne utilizando el protocolo de sincronización de la ovulación del estudio se puede realizar la IATF entre las 48 y $56 \mathrm{~h}$ pos-retiro del dispositivo sin afectar el porcentaje de preñez.

Palabras clave: dispositivo intravaginal con progesterona; cipionato de estradiol; hora de IATF; IATF

${ }^{1}$ Área de Reproducción, FISFARVET, Centro de Investigación Veterinaria de Tandil (CIVETAN, CONICET-CICPBA), Universidad Nacional del Centro de la Provincia de Buenos Aires, Tandil, Buenos Aires, Argentina

${ }^{2}$ Facultad de Ciencias Veterinarias, Universidad Nacional del Centro de la Provincia de Buenos Aires, Tandil, Buenos Aires, Argentina

${ }^{3}$ E-mail: callejas@vet.unicen.edu.ar

Recibido: 15 de octubre de 2017

Aceptado para publicación: 2 de marzo de 2018 
In the study was used 33315 -month-old beef heifers with body condition of 6 on average (scale 1-9). On this, 53.5\% presented an active corpus luteum at the beginning of the study. On day 0 , an intravaginal device with $0.5 \mathrm{~g}$ of progesterone (IVD) was placed and $2 \mathrm{mg}$ of estradiol benzoate was injected. On day 8 the IVD was removed and $0.5 \mathrm{mg}$ of cloprostenol and $0.5 \mathrm{mg}$ of estradiol cypionate were i.m. injected. On day 10 , artificial insemination at fixed time (IATF) was carried out, distributing the animals at random to be inseminated in three-time ranges: I) $48-50 \mathrm{~h}$; II: $>50-53 \mathrm{~h}$; III) $>53-56$ h. Frozen semen in 0.5 $\mathrm{ml}$ straws was used. Pregnancy diagnosis was performed by ultrasound at 30 days postIATF. Two animals lost the DISP and were excluded from the analysis. There was no effect on pregnancy rate of the time range in which the IATF was performed after the DISP was removed (I: $64.0 \%$, II: 69.8\%, III: 66.9\%). It is concluded that in beef heifers using the ovulation synchronization protocol of the study, the IATF can be performed between 48 and $56 \mathrm{~h}$ after the removal of the device without affecting the pregnancy rate.

Key words: progesterone intravaginal device; estradiol cipionate; FTAI time; FTAI

\section{INTRODUCCIÓN}

El manejo de los ciclos estruales, para que todas las hembras ovulen en un periodo breve, es el objetivo que ha estimulado el desarrollo de numerosas líneas de investigación a través de los años. Estas investigaciones, sobre todo las realizadas en las últimas dos décadas, llevaron al diseño de protocolos que permiten realizar la inseminación artificial (IA) sin la necesidad de detectar el celo, modalidad denominada comúnmente IA a tiempo fijo (IATF) (Bó et al., 2016).

En Argentina se ha favorecido la adopción del servicio de 15-17 meses de edad en numerosos rodeos de cría bovina de la pampa húmeda (Chayer et al., 2010), con la finalidad de lograr un ingreso más rápido de la hembra de reposición al circuito productivo que posibilite obtener un mayor número de terneros en la vida útil de la vaca y, en consecuencia, un aumento en la producción de carne por hectárea.

En general, los protocolos de IATF se pueden dividir en aquellos que utilizan combinaciones de $\mathrm{GnRH}$ y prostaglandina $\mathrm{F}_{2 \mathrm{a}}$
(PGF), llamados Ovsynch y los que emplean dispositivos con progesterona $\left(\mathrm{P}_{4}\right)$ y estradiol (Lane et al., 2008). En los animales con actividad sexual cíclica, la administración de progesterona tiene por finalidad mantener una fase luteal artificial hasta que la regresión del cuerpo lúteo haya ocurrido en todos los animales, de modo que al retirar el tratamiento exógeno se produce una fase preovulatoria simultánea en todos los animales tratados (Callejas, 2005).

Los estrógenos aplicados al día 0 del protocolo, juntamente con la progesterona que contiene el dispositivo intravaginal (DISP) han sido utilizados para controlar y sincronizar la onda de crecimiento folicular (Bo et al., 1995; Burke et al., 1999).

La aplicación de PGF o sus análogos en el día de extracción del DISP provoca la lisis de algún cuerpo lúteo que pudiera existir, con el consiguiente descenso de los niveles plasmáticos de progesterona (Odde, 1990). Por último, la inyección de estrógenos a las 24 horas posteriores al retiro de los DISP provoca la sincronización de los celos (Lane et al., 2001). 
La administración de cipionato de estradiol (CPE) al momento de retirar los DISP, en reemplazo de la inyección de benzoato de estradiol (BE) que se aplica 24 horas más tarde, permite evitar un encierre de los animales sin que se vea afectado el porcentaje de preñez (Sales et al., 2012). Con relación al uso de este protocolo, tiene particular importancia el rango horario en el que se puede realizar la IATF luego del retiro de los DISP para no afectar la fertilidad. El tema reviste sumo interés dado que define la elasticidad del tratamiento a la hora de planificar el número de animales a inseminar. El objetivo del presente trabajo fue evaluar en vaquillonas Bos taurus destinadas a la producción de carne el efecto del rango horario en el que se realiza la IATF sobre el porcentaje de preñez cuando son tratadas a los 15 meses de edad con un DISP combinado con estrógenos y PGF.

\section{MATERIALES y Métodos}

\section{Lugar y Animales}

El ensayo se llevó a cabo en un establecimiento agropecuario ubicado en el partido de General Alvear, Provincia de Buenos Aires, Argentina. Se utilizaron 333 vaquillonas Bos taurus, con una condición corporal promedio de 6 (escala de 1 a 9; Wagner et al., 1998 ), peso promedio de $294.6 \pm 25.5 \mathrm{~kg}$, un frame score (medida que estima el tamaño corporal del animal) de $3.6 \pm 0.7$ y un área pélvica de $151.9 \pm 9.5 \mathrm{~cm}^{2}$.

Los animales fueron destetados en marzo en el mismo establecimiento y la alimentación durante la recría fue exclusivamente pastoril a base de pasturas asociadas (festuca - Festuca arundinacea, lotus - Lotus tenuis, trébol blanco - Trifolium repens) y verdeos de invierno (promociones de rye grass - Lolium perenne).
Durante la ejecución del experimento se tuvieron en cuenta las regulaciones del Comité de Bienestar Animal de la Facultad de Ciencias Veterinarias, UNCPBA, Tandil, Buenos Aires, Argentina.

\section{Protocolo de Sincronización e Insemina- ción}

Se colocó un dispositivo intravaginal con $0.5 \mathrm{~g}$ de progesterona (DIB; Zoetis, Argentina) y se inyectó $2 \mathrm{mg}$ benzoato de estradiol i.m. en el día 0 del experimento. El 53.5\% presentaba cuerpo lúteo activo. Al día 8 , se retiró el DISP y se inyectó $0.5 \mathrm{mg}$ de cloprostenol (Ciclase DL, Zoetis) y $0.5 \mathrm{mg}$ de CPE (Cipiosin, Zoetis) i.m. Esta última actividad se desarrolló en un lapso de 2 h 58 min (entre las 09:37 y las 12:35 h)

El día 10 se llevó a cabo la IATF, utilizándose semen congelado/descongelado en pajuelas de $0.5 \mathrm{ml}$, proveniente de un toro de probada fertilidad. Para esto, los animales en que el retiro del DIB se hizo entre las 09:37 y las 11:00 se inseminaron entre las 10:36 y las 13:42 y aquellos donde el retiro se hizo entre las 11:01 y las 12:35 el servicio tuvo lugar entre las 16:08 y las 18:51. Es importante resaltar que entre las 13:42 y las 16:08 no se realizaron inseminaciones con el fin de obtener la amplitud horaria deseada. De esta manera quedó establecido un rango horario de retiro del DISP y la IATF comprendido entre 48 y 56 h. Para el análisis de la información, dentro de dicho rango se conformaron tres grupos: I, $48-50 \mathrm{~h}$; II, $>50-53 \mathrm{~h}$; III, $>53-56 \mathrm{~h}$. Todas las inseminaciones fueron realizadas por el mismo técnico.

\section{Diagnóstico de Gestación}

El diagnóstico de gestación se realizó mediante ultrasonografía (Ecógrafo Mindray DP-6600Vet, transductor transrectal lineal electrónico 75L50EAV, multifrecuencia de 5.0-10 MHz) a los 30 días de la IATF. 
Cuadro. 1. Porcentaje de preñez en vaquillonas de 15 meses tratadas con un dispositivo intravaginal con progesterona, cipionato de estradiol y prostaglandina administrados en el momento de retirar el dispositivo e inseminación a tiempo fijo en tres rangos horarios

\begin{tabular}{ccc}
\hline Horas & $\begin{array}{c}\text { Vaquillonas } \\
(\mathrm{n})\end{array}$ & $\begin{array}{c}\text { Preñez } \\
(\%)\end{array}$ \\
\hline $48-50$ & 75 & 64.0 \\
$>50-53$ & 129 & 69.8 \\
$>53-56$ & 127 & 66.9 \\
\hline Total & 331 & 67.4 \\
\hline
\end{tabular}

\section{Análisis Estadístico}

Dos animales perdieron el DISP y fueron retirados del análisis estadístico. Se evaluó el efecto del rango horario sobre el porcentaje de preñez a la IATF, utilizando el subprograma CATMOD, del paquete estadístico SAS. Se fijó un nivel de confianza del $95 \%(\alpha=0.05)$.

\section{Resultados}

No se observó efecto del rango horario en el que se realizó la IATF luego de retirado el dispositivo intravaginal con progesterona sobre el porcentaje de preñez (Cuadro 1).

\section{Discusión}

La pérdida de dispositivos fue del $0.6 \%$, encontrándose en el rango inferior citado en la literatura que varía entre 0 y $12.5 \%$ (Folman et al., 1983).

Con respecto al rango horario en que se realizó la IATF, Mechaca et al. (2013) informan que se puede realizar entre las $46 \mathrm{y}$
$58 \mathrm{~h}$ sin afectar el porcentaje de preñez. Estos investigadores trabajaron con hembras de diferentes categorías, registrando porcentajes de preñez en vaquillonas del $57.8 \%$ y $57.5 \%$ y en vacas de 53.5 y $53.9 \%$, según fuera la IATF a las 48 o $54 \mathrm{~h}$ de retirado los dispositivos, respectivamente. Estos resultados son coincidentes con los registrados en el presente trabajo en cuanto a la elasticidad en que se puede realizar la IATF sin afectar el porcentaje de preñez.

El porcentaje de preñez observado en este trabajo $(67.4 \%)$ fue muy bueno, encontrándose por encima de la media del 50.0\% citada en la bibliografía (Bo et al., 2016). Estos autores, asimismo, indican que en el $24 \%$ de los rodeos se obtienen porcentajes de preñez que varían entre el 60 y el 70\%.

\section{Conclusión}

En vaquillonas Bos taurus destinadas a la producción de carne, tratadas a los 15 meses de edad con un dispositivo intravaginal con $0.5 \mathrm{~g}$ de progesterona, $2 \mathrm{mg}$ de benzoato de estradiol inyectable y una dosis de $0.5 \mathrm{mg}$ de cloprostenol más $0.5 \mathrm{mg}$ de cipionato de estradiol al momento de retirar el dispositivo intravaginal, se puede realizar la inseminación artificial a tiempo fijo entre las 48 y $56 \mathrm{~h}$ de retirados los dispositivos sin afectar el porcentaje de preñez. Esto permite contar con cierta elasticidad en el tratamiento a la hora de planificar el número de animales a inseminar.

\section{Literatura Citada}

\section{Bo G, Adansm G, Caccia, M, Pierson} $\boldsymbol{R}$, Mapletoft R. 1995. Exogenous control of follicular wave emergence in cattle. Theriogenology 43: 31-40. doi: 10.1016/0093-691X(94)00010-R

2. Burke C, Boland M, Macmillan $K$. 1999. Ovarian responses to progesterone and oestradiol benzoate administrated 
intravaginally during dioestrus in cattle. Anim Reprod Sci 55: 23-33.

3. Bó A, de la Mata, J, Barusellis P, Menchaca A. 2016. Alternative programs for synchronizing and resynchronizing ovulation in beef cattle. Theriogenology 86: 388-396. doi: 10.1016/j.theriogenology.2016.04.053

4. Callejas S. 2005. Control farmacológico del ciclo estral: bases fisiológicas, protocolos y resultados. Parte II. Rev Taurus 25(7): 16-35.

5. Chayer R, González Chaves $S$, Villa J, Callejas S. 2010. Claves para el manejo de las vaquillonas de reposición: uso de herramientas de evaluación para la selección de vaquillonas que recibirán servicio a los 15-17 meses de edad. En: Mem V Jornadas Taurus de Reproducción Bovina. Argentina.

6. Folman Y, McPhee SR, Cumming IA, Davis IF, Chamley WA. 1983. Conception rates in cows after various synchronisaton techniques using progesterone realizing intravaginal devices. Aust Vet J 60: 44-47. doi: 10.1111/j.1751-0813.1983.tb05860.x

7. Lane E, Austin E, Crowe M. 2008. Oestrous synchronisation in cattle current options following the EU regulations restricting use of oestrogenic compounds in food-producing animals: a review. Anim Reprod Sci 109: 1-16. doi: 10.1016/j.anireprosci.2008.08.009
8. Lane E, Austin E, Roche J, Crowe M. 2001. The effect of estradiol benzoate on synchrony of estrous and fertility after removal of a progesterone-releasing intravaginal device. Theriogenology 55: 1807-1818. doi: 10.1016/S0093691X(01)00523-4

9. Menchaca A, Nuñez $R$, de Castro T, García Pintos C, Cuadro F. 2013. Implementación de programas de IATF en rodeos de cría. Seminario de actualización técnica: cría vacuna. Uruguay: Serie Técnica 208. INIA. p 229-246.

10. Odde $K, 1990$. A review of synchronization of estrus in postpartum cattle. J Anim Sci 68: 817-830.

11. Sales JN, Carvalho JB, Crepaldi GA, Cipriano RS, Jacomini JO, Maio JR, Souza JC, et al. 2012. Effects of two estradiol esters (benzoate and cypionate) on the induction of synchronized ovulations in Bos indicus cows submitted to a timed artificial insemination protocol. Theriogenology 78: 510-516. doi: 10.1016/j.theriogenology.2012.02.031

12. Wagner JJ, Lusby KS, Oltjen JW, Rakestraw J, Wettemann RP, Walters LE. 1998. Carcass composition in mature Hereford cows: estimation and effect on daily metabolizable energy requirement during winter. J Anim Sci 66: 603-612. 